\title{
SPACES WITH COARSER MINIMAL HAUSDORFF TOPOLOGIES
}

BY

\author{
JACK PORTER AND JOHANNES VERMEER
}

\begin{abstract}
A technique is developed, using $H$-closed extensions, for determining when certain Hausdorff spaces are Katetov, i.e., have a coarser minimal Hausdorff topology. Our technique works for Cech-complete Lindelöf spaces, complete metrizable spaces, and many other spaces. Also, a number of interesting examples are presented; the most striking is an example of a Katetov space whose semiregularization is not Katetov.
\end{abstract}

1. Introduction and preliminaries. A space is called Katetov, see [BPS, G], if it has a coarser minimal Hausdorff topology. In 1941, Bourbaki [B] proved that the space $\mathbf{Q}$ of rational numbers does not have a coarser compact topology but could not determine whether $\mathbf{Q}$ is Katetov. In 1965, Herrlich [He] showed that $\mathbf{Q}$ is not Katetov.

Since the semiregularization of an $H$-closed topology is a coarser minimal Hausdorff topology, it easily follows that a space is Katetov iff it has a coarser $H$-closed topology. One of the major unsolved problems in the theory of $H$-closed spaces is to find an internal characterization of Katetov spaces. In this paper, tools are developed and used to show that, surprisingly, large classes of spaces are Katetov. In particular, regular, Čech-complete Lindelöf spaces and complete metric spaces are Katetov; both of these results extend a result by Gryzlov [G]. An example of a Katetov space is given with the most interesting property that its semiregularization is not Katetov. A space is shown to be Katetov if its absolute is Katetov; however, the converse is shown to be false. A countable Katetov space is shown to be scattered. If $Y$ is a compact, extremally disconnected space without isolated points and $S$ is a countable discrete subspace of $Y$, then the complete, extremally disconnected space $Y \backslash S$ is shown not to be Katetov.

We thank the referee for his or her comments.

Now, some definitions and preliminary results needed in the sequel are presented. All spaces considered in the paper are Hausdorff.

The topology of a space $X$ is denoted by $\tau(X)$, and the semiregularization of $X$ is denoted by $X_{s}$. A coarser topology $\sigma$ on $X$, i.e., $\sigma \subseteq \tau(X)$, is sometimes called a subtopology of $\tau(X)$. The basic results about $H$-closed and minimal Hausdorff spaces can be found in [BPS, $\mathbf{P V} \mathbf{V}_{\mathbf{1}}, \mathbf{P V}_{\mathbf{2}}$ ].

For spaces $X$ and $Y$, a function $f: X \rightarrow Y$ is $\Theta$-continuous if for each $x \in X$ and open neighborhood $U$ of $f(x)$, there is an open neighborhood $V$ of $x$ such that $f\left[\operatorname{cl}_{X} V\right] \subseteq \operatorname{cl}_{Y} U$, is irreducible if $f[A] \neq Y$ whenever $A$ is a proper closed subspace of $X$, is compact if $f^{-1}(y)$ is compact for all $y \in Y$, and is perfect if $f$ is compact

Received by the editors June 4, 1984.

1980 Mathematics Subject Classification. Primary 54D25; Secondary 54D30, 54035.

Key words and phrases. Katetov spaces, minimal Hausdorff spaces, $H$-closed extensions, absolutes.

(c) 1985 American Mathematical Society $0002-9947 / 85 \$ 1.00+\$ .25$ per page 
and closed. Note that for the definitions of compact and irreducible, no topology on $Y$ is needed, that continuity is not part of the definition of perfect, and that the composition of perfect functions is perfect (see $[\mathbf{W h}]$ ). For a space $X$, recall that there is an extremally disconnected, Tychonoff space $E X$ and a perfect, irreducible, $\Theta$-continuous surjection $k: E X \rightarrow X$. The pair $E X$ and $k$ is called the absolute of $X$ and is unique in this sense: if $Y$ is an extremally disconnected, Tychonoff space and $f: Y \rightarrow X$ is a perfect, irreducible, $\Theta$-continuous surjection, then there is a homeomorphism $h: E X \rightarrow Y$ such that $f \circ h=k$. We will frequently use the facts that $X$ is $H$-closed iff $E X$ is compact and that if $g: Z \rightarrow X$ is a perfect, irreducible, $\Theta$-continuous surjection, then $E Z=E X$. Two spaces $X$ and $Z$ are said to be coabsolute if $E X=E Z$. Basic results about the absolutes of spaces are contained in the excellent survey [Wo].

Now, some of the tools to determine when a space is Katetov are presented. Let $Y$ be a space, $X$ a set, and $f: Y \rightarrow X$ a surjection. The collection $\{f[A]: A$ is a closed subset of $Y\}$ is a closed base for a topology $\tau$ (not necessarily Hausdorff) on $X$; the topology $\tau$ is called the $\Theta$-quotient topology on $X$.

1.1. THEOREM [R, VW]. Let $Y$ be a compact space, $X$ a set, and $f: Y \rightarrow X$ a compact and irreducible surjection. The $\Theta$-quotient topology $\tau$ on $X$ is minimal Hausdorff and $f: Y \rightarrow(X, \tau)$ is perfect and $\Theta$-continuous.

Note that in the setting of 1.1 , the $\Theta$-quotient topology coincides with the quotient topology iff the quotient topology is Hausdorff. Additional information about the $\Theta$-quotient topology can be found in $\left[\mathbf{B L}, \mathbf{V}_{\mathbf{1}}, \mathbf{V}_{\mathbf{2}}\right]$.

For a space $X, A \subseteq X$ is $\Theta$-closed if for each $p \in X \backslash A$, there is a neighborhood $U$ of $p$ such that $\operatorname{cl}_{X} U \cap A=\varnothing$. By 3.4 in $\left[\mathbf{D P}_{\mathbf{1}}\right]$, if $B \subseteq X$, then $k^{-1}[B]$ is closed where $k: E X \rightarrow X$ is described above iff $B$ is $\Theta$-closed.

Our next result is a major tool that is frequently used throughout the sequel.

1.2. THEOREM. For a space $X$, the following are equivalent:

(a) $X$ is Katetov.

(b) $X$ is the perfect image of a compact space.

(c) $X$ is the perfect image of an $H$-closed space.

(d) There are an $H$-closed space $Y$ and a closed surjection $f: Y \rightarrow X$ such that $f^{-1}(x)$ is $\Theta$-closed for each $x \in X$.

Proof. The proofs of (b) implies (c) and (c) implies (d) are easy. To prove (a) implies (b), let $X$ be a Katetov space and $\tau$ a minimal Hausdorff subtopology of $\tau(X)$. Let $Y=E(X, \tau)$. Then $Y$ is compact and since the function $k: Y \rightarrow(X, \tau)$ is perfect and $\tau \subseteq \tau(X)$, then $k: Y \rightarrow X$ is also perfect. Finally, to prove (d) implies (a), let $Y$ be an $H$-closed space and $f: Y \rightarrow X$ be closed surjection such that $f^{-1}(x)$ is $\Theta$-closed for each $x \in X$. Now, $E Y$ is compact and $k: E Y \rightarrow Y$ is perfect. Since, by 3.4 in $\left[\mathbf{D P}_{\mathbf{1}}\right], k^{-1}\left[f^{-1}(x)\right]$ is compact for each $x \in X$, it follows that $f \circ k$ is perfect. By a result in $[\mathbf{S}]$, there is a closed set $D \subseteq E Y$ such that $\left.f \circ k\right|_{D}: D \rightarrow X$ is an irreducible surjection. Since $\left.f \circ k\right|_{D}$ is compact, by 1.1 there is a minimal Hausdorff topology $\tau$ on $X$. Since $\left.f \circ k\right|_{D}$ is closed, $\tau \subseteq \tau(X)$. Hence $X$ is a Katetov space.

1.3. COROLlaRY. The perfect image of a Katetov space is a Katetov space. 
Proof. Let $Y$ be a perfect image of a Katetov space $X$ by a function $f$. By $1.2, Y$ is the perfect image of some compact space $Z$ by a function $g$. Thus, $X$ is the perfect image of $Z$ by $f \circ g$. So, by $1.2, X$ is a Katetov space.

An example is given in the second section which shows that the perfect, irreducible, $\Theta$-continuous preimage of a Katetov space is not necessarily Katetov.

1.4. COROLlaRY. If $X$ has an $H$-closed extension $h X$ and a closed discrete subspace $A$ such that $|h X \backslash X| \leq|A|$, then $X$ is a Katetov space.

Proof. Let $f: h X \backslash X \rightarrow A$ be a one-to-one function. Define $g: h X \rightarrow X$ by $g(x)=x$ for $x \in X$ and $g(p)=f(p)$ for $p \in h X \backslash X$. Since $g^{-1}(y)$ is finite for all $y \in X, g$ is compact. Let $B$ be a closed subset of $h X$. Since $g[B]=(B \cap X) \cup f[B \backslash X]$, $f[B \backslash X] \subseteq A$, and every subset of $A$ is closed in $X$, it follows that $g[B]$ is closed. By $1.2, X$ is a Katetov space.

The result of 1.4 gives a technique for constructing Katetov spaces, e.g., noncountably compact spaces which have $H$-closed extensions with countable remainders. So, for an infinite cardinal $\alpha$, those spaces $X$ with $H$-closed extensions $h X$ such that $|h X \backslash X| \leq \alpha$ will be of interest. If $\sigma X$ is the Fomin $H$-closed extension of a space $X$ (see $\left[\mathbf{F}, \mathbf{P V}_{\mathbf{1}}, \mathbf{P V _ { 2 }}\right]$ ), then by 0.5 in [Po], $X$ has an $H$-closed extension $h X$ such that $|h X \backslash X|=\alpha$ iff there is a partition $P$ of $\sigma X \backslash X$ into compact sets such that $|P|=\alpha$. By 5.10 in $\left[\mathbf{P V}_{2}\right], \sigma X \backslash X=\beta E X \backslash E X$; so there is an $H$-closed extension $h X$ of $X$ such that $|h X \backslash X|=\alpha$ iff there is a partition $P$ of $\beta E X \backslash E X$ into compact subsets such that $|P|=\alpha$.

Another external characterization of Katetov spaces is provided in $\left[\mathbf{D P}_{\mathbf{2}}, \mathbf{V}_{\mathbf{2}}\right.$ ] and is included here for completeness.

1.5. THEOREM. A space is Katetov iff it is the remainder of some $H$-closed extension of a discrete space.

As previously noted, the space $\mathbf{Q}$ is not Katetov; the same type of proof yields the next result.

1.6. THEOREM. A Katetov space is not the countable union of compact, nowhere dense sets.

An example in the second section (the space described in 2.4) shows that even among Urysohn spaces, the "compactness" in 1.6 cannot be replaced by " $H$-closed and $\Theta$-closed".

2. Relationship among the subtopologies of $X, X_{s}$, and $E X$. Let $X$ be a space. If one of the three spaces $X, X_{s}$, or $E X$ has a compact (resp. minimal Hausdorff) subtopology, we are interested when the other two have a compact (resp. minimal Hausdorff) subtopology. Of course, if $X_{s}$ has a compact or minimal Hausdorff subtopology, then so does $X$.

2.1. Proposition. A regular subtopology of a space $X$ is a subtopology of $X_{s}$.

Proof. Let $\tau$ be a regular subtopology of $X$ and $A$ a closed subset of $(X, \tau)$. Then $A$ is $\Theta$-closed in $(X, \tau)$ and, hence, $A$ is $\Theta$-closed in $X$. It follows that $A$ is closed in $X_{s}$ and $\tau \subseteq \tau\left(X_{s}\right)$. 
2.2. COROLlary. A space $X$ has a compact subtopology iff $X_{s}$ has a compact subtopology.

It is not true that if $X$ is Katetov, then $X_{s}$ is also Katetov. Before establishing this fact, a theorem which generalizes a result by Gryzlov $[\mathbf{G}]$ is needed.

2.3. THEOREM. If $h X$ is an $H$-closed extension of a space $X$ such that $h X \backslash X$ is closed and discrete, then $X \times h X$ is a Katetov space.

Proof. Fix $p \in X$. Define a function $f: h X \times h X \rightarrow X \times h X$ by $f(x, y)=(x, y)$ for $(x, y) \in X \times h X$ and $f(z, y)=(p, z)$ for $(z, y) \in(h X \backslash X) \times h X$. Then $f$ is closed (see the proof of 1.4). For $(x, y) \in X \times h X, f^{-1}(x, y)=\{(x, y)\}$ if $x \neq p$ or $y \in X$, and $f^{-1}(p, y)=\{(p, y)\} \cup\{y\} \times h X$ if $y \in h X \backslash X$. It is easy to verify that $f^{-1}(x, y)$ is $\Theta$-closed in $h X \times h X$ for each $(x, y) \in X \times h X$. By 1.2, it follows that $X \times h X$ is Katetov.

Now, an example of a Katetov space $X$ is given such that $X_{s}$ is not Katetov.

2.4. EXAMPLE. Let $\mathbf{R}^{*}$ be the one-point compactification of the space $\mathbf{R}$ of real numbers. Then $\mathbf{R}^{*}$ is a compactification of $\mathbf{Q}$. Let $h \mathbf{Q}$ be $\mathbf{R}^{*}$ with this topology:

$U \subseteq \mathbf{R}^{*}$ is open in $h \mathbf{Q}$ iff $p \in U$ implies there is some $V \in \tau\left(\mathbf{R}^{*}\right)$ such that $p \in V$ and $V \cap \mathbf{Q} \subseteq U$.

Now, $h \mathbf{Q}$ is the simple extension (see $\left[\mathbf{P} \mathbf{V}_{\mathbf{1}}\right]$ ) of $\mathbf{R}^{*}$, and it is easy to verify that $h \mathbf{Q}$ is an $H$-closed extension of $\mathbf{Q}$ such that $h \mathbf{Q} \backslash \mathbf{Q}$ is closed and discrete and $(h \mathbf{Q})_{s}=\mathbf{R}^{*}$. By $2.3, X=\mathbf{Q} \times h \mathbf{Q}$ is a Katetov space. However, $X_{s}=(\mathbf{Q} \times h \mathbf{Q})_{s}=$ $\mathbf{Q}_{s} \times(h \mathbf{Q})_{s}=\mathbf{Q} \times \mathbf{R}^{*}$ is not Katetov by $\mathbf{1 . 6}$.

2.5. REMARKS. Let $X$ be the space described in 2.4 .

(a) The space $X$ shows that "regularity" in 2.1 cannot be replaced by "semiregularity".

(b) Note that the space $X$ is Urysohn, i.e., every pair of distinct points can be separated by disjoint closed neighborhoods, and $X=\bigcup\{\{r\} \times h \mathbf{Q}: r \in \mathbf{Q}\}$ is the countable union of nowhere dense $H$-closed and $\Theta$-closed subspaces. So, the result of 1.6 is not true when "compactness" is replaced by " $H$-closed and $\Theta$-closed", even among Urysohn spaces.

(c) Since the identity function from $X_{s}$ to $X$ is a $\Theta$-continuous, perfect, irreducible bijection, it follows that "image" in 1.3 cannot be replaced by "preimage".

Let $X$ be a space. We now turn our interest to when one of the spaces $X$ or $E X$ has a compact (resp. minimal Hausdorff) subtopology implies that the other space has a compact (resp. minimal Hausdorff) subtopology. The next result is an immediate corollary of 1.3 .

2.6. Proposition. If the absolute EX of a space $X$ is Katetov, then so is the space.

Let $X$ be the Katetov space described in 2.4. Since $E X=E X_{s}$ and $X_{s}$ is not Katetov, then $E X$ is not a Katetov space by 2.6 even though $X$ is a Katetov space.

If $X$ is a minimal Hausdorff space which is not compact, then $E X$ is compact and $X$ has no coarser compact subtopology. Now, we give examples of spaces $X$ and $Y$ such that $X$ has no coarser compact topology, $Y$ has a coarser compact subtopology, and yet, $E X=E Y$. Before presenting this result, another fact is needed. 
2.7. Proposition. If $X$ is a space which is locally compact except for at most one point, then $X$ has a compact subtopology.

PROOF. The proof is straightforward.

2.8. EXAMPLE. Consider the following subspace

$$
X=\bigcup\{(1 / n, y): 0 \leq y \leq 1, n \in \mathbf{N}\} \cup\{(0,0),(0,1)\}
$$

of $\mathbf{R}^{2}$. By a result in $[\mathbf{P a}], X$ has no compact subtopology. Let

$$
\begin{gathered}
Y_{1}=\operatorname{cl}_{X}(\bigcup\{(\bigcup\{(1 / n, y): n \in \mathbf{N}, 1 / 2 \leq y \leq 1\})\}), \\
Y_{2}=\operatorname{cl}_{X}(\bigcup\{(1 / n, y): n \in \mathbf{N}, 0 \leq y \leq 1 / 2\})
\end{gathered}
$$

and $Y=Y_{1} \oplus Y_{2}$. Then the function $f: Y \rightarrow X$ defined by $f(p, i)=p$ for $i=1,2$, is a perfect, irreducible continuous surjection. So, by the Fundamental Theorem of Absolutes, $E X=E Y$. By 2.7, $Y_{1}$ and $Y_{2}$ have compact subtopologies. Hence, $Y$ has a compact subtopology.

When $Y$ is the space described in 2.8, we do not know if $E Y$ has a coarser compact subtopology, or not. However, in 5.4, we construct a zero-dimensional space $X$ with a compact subtopology such that $E X$ is not Katetov.

3. $H$-closed extensions with countable remainders. By 1.4 , a space $X$ is Katetov if $X$ has an infinite closed discrete subset, i.e., $X$ is not countably compact, and $X$ has an $H$-closed extension with a countable remainder. As noted in the paragraph following 1.4, a space $X$ has an $H$-closed extension with a countable remainder if $\beta E X \backslash E X$ has a countable partition of compact sets. Since $\beta E X \backslash E X$ is zero-dimensional, the next result, communicated to us by F. Galvin, is quite useful.

3.1. Proposition. A zero-dimensional space $Y$ can be partitioned into a countable number of compact sets iff $Y$ is the countable union of compact $G_{\delta}$-sets.

PROOF. If $Y$ can be partitioned into a countable number of compact sets, then clearly each of the compact sets is $G_{\delta}$. Conversely, suppose $Y=\bigcup\left\{A_{n}: n \in \mathbf{N}\right\}$, where each $A_{n}$ is a compact, $G_{\delta}$-set. Since the finite union of compact $G_{\delta}$-sets is a compact $G_{\delta}$-set, we will assume that $A_{n} \subseteq A_{n+1}$ for $n \in \mathbf{N}$. Using that $Y$ is zero-dimensional, it follows that for each $n \in \mathbf{N}, A_{n}=\bigcap\left\{C_{m}^{n}: m \in \mathbf{N}\right\}$, where $C_{m}^{n}$ is clopen, $C_{1}^{n}=X$, and $C_{m}^{n} \supseteq C_{m+1}^{n}$ for $m \in \mathbf{N}$. Let $D_{n}^{m}=A_{n+1} \cap\left(C_{m}^{n} \backslash C_{m+1}^{n}\right)$ for $n, m \in \mathbf{N}$. Then $P=\left\{A_{1}\right\} \cup\left\{D_{n}^{m}: n, m \in \mathbf{N}\right\} \backslash\{\varnothing\}$ is a countable partition of compact subsets of $Y$.

If $X$ is a Tychonoff space, the subspace $X \cap \operatorname{cl}_{\beta X}(\beta X \backslash X)$ is called the residue of $X$ and is denoted by $R(X)$. Note that $X \backslash R(X)=\{p \in X: X$ has a compact neighborhood of $p$ \}, that $R(X)=X \cap \operatorname{cl}_{Y}(Y \backslash X)$ for any compactification $Y$ of $X$, and that $R(X)$ is closed. Recall that $X$ is called $\check{C}_{\text {ech-complete }}$ if $X$ is a $G_{\delta}$-subset of $\beta X$, or equivalently, if $c X \backslash X$ is a $\sigma$-compact subspace for every compactification $c X$ of $X$.

3.2. PROPOSITION. If $c X$ is a zero-dimensional compactification of a Cechcomplete space $X$ and $R(X)$ is Lindelöf, then $c X \backslash X$ has a countable partition of compact sets. 
Proof. Since $c X \backslash X$ is a $\sigma$-compact space, $c X \backslash X=\bigcup\left\{A_{n}: n \in \mathbf{N}\right\}$, where $A_{n}$ is compact for each $n \in \mathbf{N}$. Let $Y=\operatorname{cl}_{c X}(c X \backslash X)$. Fix $n \in \mathbf{N}$. For $p \in R(X)$, there is a clopen set $U_{p}$ in $Y$ such that $p \in U_{p}$ and $U_{p} \cap A_{n}=\varnothing$. There is a countable subset $F \subseteq R(X)$ such that $R(X) \subseteq U_{n} \subseteq Y \backslash A_{n}$, where $U_{n}=\bigcup\left\{U_{p}: p \in F\right\}$. If $B_{n}=Y \backslash U_{n}$, then $A_{n} \subseteq B_{n} \subseteq c X \backslash X, B_{n}$ is a compact $G_{\delta}$-set, and $c X \backslash X=$ $\bigcup\left\{B_{m}: m \in \mathbf{N}\right\}$. By $3.1, c X \backslash X$ has a countable partition of compact sets.

\subsection{COROLlary. Let $X$ be a space.}

(a) If $X$ is not countably compact, $E X$ is Čech-complete, and $R(E X)$ is Lindelöf, then $X$ has an $H$-closed extension with a countable remainder and is Katetov.

(b) If $X$ is Tychonoff and Cech-complete and $R(X)$ is Lindelof, then $X$ has an $H$-closed extension with a countable remainder.

PROOF. Part (a) follows from 3.2 and the results stated in the paragraph before 3.1. Part (b) follows from 3.2 and these facts: (i) if $X$ is Cech-complete, so is $E X$, (ii) if $R(X)$ is Lindelöf, then so is $k^{-1}[R(X)]$, and (iii) $R(E X)$ is a closed subset of $k^{-1}[R(X)]$.

Now, we use 3.3(b) to establish nice conditions for a space to be Katetov.

3.4. THEOREM. Suppose $X$ is a space which satisfies

(a) $X$ is regular, Lindelöf, and Cech-complete or

(b) $X$ is paracompact, Cech-complete and $R(X)$ is Lindelöf.

Then $X$ is Katetov and has an $H$-closed extension with a countable remainder.

Proof. Both (a) and (b) satisfy the hypothesis of 3.3(b); hence, $X$ has an $H$ closed extension with a countable remainder. If $X$ is countably compact, then $X$ is compact as $X$ is Lindelöf or paracompact; so, $X$ is Katetov. If $X$ is not countably compact, then $X$ has an infinite closed discrete subspace. By the comments in the paragraph before $3.1, X$ is Katetov.

We now look at some necessary conditions for certain classes of spaces to have $H$-closed extensions with countable remainders.

3.5. LEMMA. The countable intersection of $\sigma$-compact subspaces in a regular space is Lindelöf.

Proof. Let $X$ be a regular space, $B_{n} \subseteq X$ where $B_{n}$ is $\sigma$-compact for $n \in \omega$, and $A=\bigcap\left\{B_{n}: n \in \omega\right\}$. By $3.8(\mathrm{~g})$ in $[\mathbf{E}], \prod\left\{B_{n}: n \in \omega\right\}$ is Lindelöf. The function $e: A \rightarrow \prod\left\{B_{n}: n \in \omega\right\}$ defined by $e(x)(n)=x$ is an embedding and $e[A]$ is closed in $\prod\left\{B_{n}: n \in \omega\right\}$. So, $A$ is Lindelöf.

3.6. PROPOSITION. Let $X$ be a Tychonoff space. The following are equivalent:

(a) $X$ is Lindelöf and Cech-complete.

(b) $\beta X \backslash X$ is the countable union of compact sets which are $G_{\delta}$ in $\beta X$.

(c) $\beta E X \backslash E X$ can be partitioned into a countable number of compact sets which are $G_{\delta}$ in $\beta E X$.

Proof. The proof that (a) implies (b) is similar to the proof of 3.1. Suppose (b) is true. Since $k_{\beta X}: \beta E X \rightarrow \beta X$ is continuous and $k_{\beta X}^{-1}[\beta X \backslash X]=\beta E X \backslash E X$, $\beta E X \backslash E X$ is the countable union of compact sets which are $G_{\delta}$ in $\beta E X$. By the proof of 3.1, $\beta E X \backslash E X$ can be partitioned into a countable number of compact sets which are $G_{\delta}$ in $\beta E X$, and (c) holds. To show (c) implies (a), suppose $\beta E X \backslash E X=$ 
$\bigcup\left\{A_{n}: n \in \omega\right\}$ where $A_{n}$ is compact and $G_{\delta}$ in $\beta E X$ for each $n \in \omega$. Thus, $\beta E X \backslash A_{n}$ is $\sigma$-compact, and by 3.5, $E X=\bigcap\left\{\beta E X \backslash A_{n}: n \in \omega\right\}$ is Lindelöf. As $k_{X}: E X \rightarrow X$ is continuous, $X$ is Lindelöf. Clearly, $X$ is Čech-complete.

Of course, 3.6(c) implies that the space $X$ has an $H$-closed extension with a countable remainder. Before presenting the next result, recall that a space $X$ is nowhere locally compact if $R(X)=X$, i.e., no point has a compact neighborhood.

3.7. Proposition. Let $X$ be a Tychonoff, nowhere locally compact space. If $X$ has an $H$-closed extension with a countable remainder, then $X$ has a dense Lindelöf subspace.

ProOF. By the comments following 1.4 and since $k_{X}: E X \rightarrow X$ is irreducible, it follows that $E X$ is nowhere locally compact and has an $H$-closed extension with a countable remainder. By continuity of $k_{X}$, it sufficies to show that $E X$ has a dense Lindelöf subspace. Now, $\beta E X \backslash E X$ has a countable partition $\left\{A_{n}: n \in \omega\right\}$ of compact subsets. Fix $n \in \omega$. For each $m \in \omega$ there is an open set $U_{m}$ in $\beta E X$ such that $A_{n} \subseteq U_{m}$ and $\left(\operatorname{cl}_{\beta E X} U_{m}\right) \cap A_{k}=\varnothing$ for $0 \leq k<n$ and $n<k \leq n+m+1$, and $\operatorname{cl}_{\beta E X} U_{m} \subseteq U_{m-1}$ if $m \geq 1$. Then $B_{n}=\bigcap\left\{\operatorname{cl}_{\beta E X} U_{m}: m \in \omega\right\}$ is compact, $\beta E X \backslash B_{n}$ is $\sigma$-compact, and $B_{n} \backslash E X=A_{n}$. If $V$ is an open set in $\beta E X$ and $V \subseteq B_{n}$, then $\operatorname{cl}_{\beta E X} V=\operatorname{cl}_{\beta E X}(V \backslash E X)$ as $\beta E X \backslash E X$ is dense in $\beta E X$. Since $V \backslash E X \subseteq B_{n} \backslash E X=A_{n}, \operatorname{cl}_{\beta E X} V \subseteq \operatorname{cl}_{\beta E X} A_{n}=A_{n}$. So $V \subseteq A_{n} \subseteq \beta E X \backslash E X$ implying that $V=\varnothing$. Hence, $\beta E X \backslash B_{n}$ is open and dense. By Baire's Theorem and 3.5, $\left\{\beta E X \backslash B_{n}: n \in \omega\right\}$ is dense in $E X$ and Lindelöf.

3.8. EXAMPLES. (a) If $D$ is a discrete space of cardinality $\aleph_{1}$, and $\mathbf{P}$ is the space of irrationals, then $D \times \mathbf{P}$ is a complete metric space with no dense Lindelöf space. By 3.7, $\mathbf{D} \times \mathbf{P}$ has no $H$-closed extension with a countable remainder.

(b) The converse of 3.7 is false as $\mathbf{Q}$ is Lindelöf, Tychonoff, and nowhere locally compact but has no $H$-closed extension with a countable remainder.

(c) If $Y$ is the one-point compactification of an uncountable discrete space, then $\mathbf{P} \times Y$ is a Cech-complete, zero-dimensional space which by Theorem 3 in $\left[\mathbf{H o}_{2}\right]$ has no compactification with a countable remainder. The space $\mathbf{P} \times Y$ has a compact subtopology, but we do not know, in general, if every Čech-complete, zerodimensional Lindelöf space must have a compact subtopology.

In $\left[\mathbf{H o}_{\mathbf{1}}\right]$, it is shown that if a paracompact space $X$ has a compactification with a countable remainder, then $R(X)$ is Lindelöf. We do not know if this statement is true when "compactification" is replaced by " $H$-closed extension". Also, we do not know if a Čech-complete, paracompact space is Katetov.

4. The metric case. In the last section, we proved that regular, Čechcomplete, Lindelöf spaces are Katetov. In this section, we show that Cech-complete metric spaces are also Katetov. Recall [E, Theorem 4.3.26] that a metric space is Cech-complete iff it is complete as a metric space. A space $X$ is strongly zerodimensional if for disjoint zero sets $Z_{1}$ and $Z_{2}$, there is a clopen set $C$ such that $Z_{1} \subseteq C \subseteq X \backslash Z_{2}$. The following result in [E] is most useful.

4.1. LEMMA. (a) [E,7.3.15] A space $X$ is strongly zero-dimensional and metrizable iff $X$ can be embedded as a subspace of $D^{\omega}$, where $D$ is a discrete space and $|D| \leq d X$. In particular, the property of being metrizable and strongly zerodimensional is both hereditary and countable productive. 
(b) $[\mathbf{F}, 7.3 \mathrm{H}]$ A completely metrizable and strongly zero-dimensional space is homeomorphic to a closed subspace of $D^{\omega}$, where $D$ is a discrete space and $|D|=$ $w X$.

To obtain the result that every complete metric space is Katetov, more preliminary results are needed.

4.2. THEOREM $[\mathbf{P y}]$. Every strongly zero-dimensional, complete metric space has a compact subtopology.

Let $I$ be the unit interval. For an infinite cardinal $\alpha$, let $X_{\alpha}$ be the topological sum of $\alpha$ copies of $I$, i.e., $X_{\alpha}=\bigoplus\left\{I_{\beta}: \beta<\alpha\right\}$, where $I_{\beta}=I \times\{\beta\}$ for $\beta<\alpha$. The hedgehog $\mathcal{H}(\alpha)$ is the quotient set of $X_{\alpha}$ where $\{0\} \times \alpha$ is identified to a point, denoted as $\overline{0}$, plus the topology (not the quotient topology) induced by this metric $d$-if $p \in I_{\beta}$ and $q \in I_{\gamma}$ for $\beta, \gamma<\alpha$, define $d(p, q)=|p-q|$ if $\beta=\gamma$ and $d(p, q)=p+q$ if $\beta \neq \gamma$; additional information about hedgehogs can be found in $[\mathbf{E}]$. Let $C$ be the Cantor subspace of $I, Y_{\alpha}=\bigoplus\left\{C_{\beta}: \beta<\alpha\right\}$ where $C_{\beta}=C \times\{\beta\}$ for $\beta<\alpha$, and $C(\alpha)$ the corresponding subspace of $\mathcal{H}(\alpha)$. By IV.4.2 in [D], there is a continuous surjection $f: C \rightarrow I$ such that $f^{-1}(0)$ is a singleton; now, $f$ is compact since $C$ is compact. The function $F: C(\alpha) \rightarrow \mathcal{H}(\alpha)$ defined by $F(x, \beta)=(f(x), \beta)$ for $x \in C$ and $\beta<\alpha$ is a perfect continuous surjection. We will use this fact to help prove the next result.

4.3. THEOREM. Every metric space $X$ is the perfect, irreducible continuous image of a strongly zero-dimensional metric space $Y$ such that $w X=w Y$ and $X$ is complete iff $Y$ is complete.

ProOF. An infinite metric space $X$ can be embedded as a subspace of $(\mathcal{H}(\alpha))^{\omega}$, where $\alpha=w X$. Let $F: C(\alpha) \rightarrow \mathcal{H}(\alpha)$ be the continuous, perfect surjection defined in the previous paragraph. If $h \in C(\alpha)^{\omega}$, i.e., $h: \omega \rightarrow C(\alpha)$ is a function, then define $G(h): \omega \rightarrow \mathcal{H}(\alpha)$ by $G(h)(n)=F(h(n))$ for $n \in \omega$. So, $G: C(\alpha)^{\omega} \rightarrow \mathcal{H}(\alpha)^{\omega}$ is a function and it follows that $G$ is a perfect, continuous surjection. Let $Z=G^{-1}[X]$ and $H=G \mid Z$. Then $H: Z \rightarrow X$ is a perfect, continuous surjection. By a result in $[\mathbf{S}]$, there is a closed subspace $Y$ of $Z$ such that $H \mid Y: Y \rightarrow X$ is a perfect, irreducible continuous surjection. Clearly, $C(\alpha)$ is a strongly zero-dimensional, metric space; by $4.1, C(\alpha)^{\omega}$ and, hence, $Y$ are strongly zero-dimensional and metrizable. Since $H \mid Y$ is perfect and irreducible, $d Y=d X$; as $X$ and $Y$ are metrizable, $w Y=d Y$ and $w X=d X$ implying $w Y=w X$. Since $H \mid Y$ is perfect, it follows that $X$ is Cech-complete if $Y$ is Cech-complete.

We now have the tools to prove one of the main results in this section.

\subsection{THEOREM. A complete metric space is Katetov.}

ProOF. By 4.3, a complete metric space $X$ is the perfect, irreducible continuous image of a strongly zero-dimensional, complete metric space $Y$. By $4.2, Y$ has a compact subtopology, and by $1.3, X$ is Katetov.

The space $X$ described in 2.8 is an example of a complete metrizable space with no compact subtopology. So, 4.4 cannot be strengthened to the statement that complete metric spaces have a coarser compact subtopology. Our next major result is to prove that spaces coabsolute with a complete metric space $X$ such that $\operatorname{cf}(d X)>\aleph_{0}$ are Katetov. Some preliminary results are needed. 
4.5. PROPOSITION. If $D$ is an infinite discrete space, then $D^{\omega}$ has a compactification such that the cardinality of the remainder is $|D|$.

PROOF. First note that the lexicographic order on $\omega_{\alpha} \times \mathbf{Z}$, where $|D|=\omega_{\alpha}$ and $\mathbf{Z}$ is the space of all integers with the discrete topology, generates the discrete topology; so, $D$ is orderable. It is straightforward to show that the topology on $D^{\omega}$ is generated by the lexicographic order $\leq$ on $D^{\omega}$ and that the remainder of the Dedekind-MacNeille completion of $\left(D^{\omega}, \leq\right)$ has cardinality $|D|$.

4.6. Proposition. (a) A strongly zero-dimensional, complete metric space $X$ has a compactification whose remainder has cardinality $\leq d X$.

(b) A space coabsolute with a complete metric space $X$ has an $H$-closed extension whose remainder has cardinality $\leq d X$.

Proof. Now, (a) is a consequence of 4.1 (b) and 4.5. To prove (b), let $X$ be a complete metric space. By $4.3, X$ is the perfect, irreducible continuous image of a strongly zero-dimensional, complete metric space $Y$ such that $d Y=d X$. In particular, $E X=E Y$. By (a), $Y$ has a compactification whose remainder has cardinality $\leq d Y$. Hence, there is a partition $P$ of $\beta E Y \backslash E Y$ into compact sets such that $|P| \leq d Y$. By the comment following 1.4, it follows that $X$ has an $H$-closed extension whose remainder is $\leq d X$ as $\beta E X \backslash E X=\beta E Y \backslash E Y$.

It is not possible to remove the hypothesis of "strongly zero-dimensional" from 4.6(a). For, if $X$ is the space described in 2.8, then $X$ is a complete metric space, $d X=\aleph_{0}$ and $X$ is not rim compact; so, by Theorem 2 in $\left[\mathbf{H o}_{\mathbf{1}}\right], X$ does not have a compactification with a countable remainder. Also, it is not possible to decrease the cardinality restriction in 4.6 (b) as the space described in 3.8(a) is a complete metric space with density $\aleph_{1}$, and has no $H$-closed extension with a countable remainder. We now present our second major result in this section.

4.7. THEOREM. If $Y$ is coabsolute with a complete metric space $X$ such that $\operatorname{cf}(d Y)>\aleph_{0}$, then $Y$ is a Katetov space.

Proof. Since $E Y=E X$ and by 1.3 , it suffices to show that $E X$ is a Katetov space. By 1.4 and 4.6, it suffices to show that $E X$ has a closed, discrete subspace of cardinality $d Y=d X$. Now, $X$ has a $\sigma$-discrete base $B=\bigcup\left\{B_{n}: n \in \omega\right\}$, where $|B|=$ $w X=d X$ and $B_{n}$ is a discrete family for each $n \in \omega$. Since $\operatorname{cf}(d X)=\operatorname{cf}(d Y)>\aleph_{0}$, then for some $n \in \omega,\left|B_{n}\right|=d X$. If $p_{A} \in A$ for each $A \in B_{n}$, then $T=\left\{p_{A}: A \in B_{n}\right\}$ is a closed discrete subspace of $X$ and $|T|=d X$. As $k_{X}: E X \rightarrow X$ is continuous, it follows that $E X$ has a closed discrete subspace of cardinality $d X$.

4.8. REMARKS. (a) Let $Y=\bigoplus\left\{H\left(\aleph_{n}\right): n \in \omega\right\}$ and $X=Y \cup\{\infty\}$, where $U \subseteq X$ is defined to be open if $U \cap Y$ is open in $Y$ and $\infty \in U$ implies for some $m \in \omega, \mathcal{H}\left(\aleph_{n}\right) \subseteq U$ for $n \geq m$. It is easy to see that $Y$ is a complete metric space, $d Y=\aleph_{\omega}$ and $Y$ has no closed discrete subspace of cardinality $\aleph_{\omega}$. So, the proof of 4.7 does not work for $Y$. For $n \in \omega$, let $y_{n}$ denote $\overline{0}$ in $H\left(\aleph_{n}\right)$ (see the notational definition in the paragraph following 4.1). Now, $E X$ is a Cech-complete, paracompact space, and since $R(E X) \subseteq \bigcup\left\{k_{X}^{-1}\left(y_{n}\right): n \in \omega\right\} \bigcup k_{X}^{-1}(\infty), R(E X)$ is $\sigma$-compact and, hence, is Lindelöf. By 3.4(b), $E X$ is Katetov. Hence, by 1.3, every space coabsolute with $X$ is Katetov.

(b) For any arbitrary complete metric space $X$, we do not know whether $E X$ has a compact subtopology or is Katetov. 
5. Additional results. In this section, we give a sufficient condition for a Tychonoff space to have a compact subtopology when a certain retract has a compact subtopology, show that a countable, Katetov space is scattered, and give examples of a complete, extremally disconnected space which is not Katetov and a zero-dimensional space with a compact subtopology such that its absolute is not Katetov.

5.1. THEOREM. Let $A$ be a retract of a Tychonoff space $X$ such that $X \backslash A$ is locally compact, and $A$ has a compact subtopology. Then $X$ has a compact subtopology.

PROOF. First, recall that retracts are closed. Let $f: X \rightarrow A$ be a retraction, and let $\tau$ be a compact subtopology of $A$. Since $f: X \rightarrow(A, \tau)$ is also continuous, there is a continuous extension $\beta f: \beta X \rightarrow(A, \tau)$. Now, define $g: \beta X \rightarrow X$ by $g(p)=p$ for $p \in X \backslash A$ and $g(p)=\beta f(p)$ for $p \in \beta X \backslash(X \backslash A)$. Since $X \backslash A$ is locally compact and open in $X$, it follows that $X \backslash A$ is open in $\beta X$ and $\beta X \backslash(X \backslash A)=(\beta X \backslash X) \cup A$ is compact. Now $g$ is a compact function as $g^{-1}(p)=p$ for $p \in X \backslash A$ and $g^{-1}(\beta)=$ $(\beta f)^{-1}(p) \cap(\beta X \backslash(X \backslash A))$ for $p \in A$. Let $\sigma$ be the quotient topology induced on $X$ by $g$; so, $(X, \sigma)$ is compact and $T_{1}$. Since $X \backslash A$ is open in $\beta X$, it follows that the identity function $g \mid(X \backslash A)$ is a homeomorphism and $X \backslash A \in \sigma$. If $B \subseteq X$ and $X \backslash B \in \sigma$, then $g^{-1}[B]$ is closed in $\beta X$ and, hence, $g^{-1}[B] \cap X=B$ is closed in $X$. Thus, $\sigma \subseteq \tau(X)$. Finally, we show that $(X, \sigma)$ is Hausdorff. Let $p$ and $q$ be distinct points of $X$. Since $X \backslash A \in \sigma$ and $g \mid(X \backslash A)$ is a homeomorphism, then if $p, q \in X \backslash A, p$ and $q$ can be separated by disjoint open neighborhoods of $\sigma$. If $p \in X \backslash A$ and $q \in A$, there is an open set $U \in \tau(X)$ such that $p \in U \subseteq \operatorname{cl}_{X} U \subseteq X \backslash A, \operatorname{cl}_{X} U$ is compact, and $q \notin \mathrm{cl}_{X} U$. Since $g^{-1}[U]=U \in \tau(\beta X)$ and $g^{-1}\left[\mathrm{cl}_{X} U\right]=\operatorname{cl}_{X} U$ is compact, it follows that $U$ and $X \backslash \operatorname{cl}_{X} U$ are disjoint open sets in $\sigma$ containing $p$ and $q$, respectively. Suppose $p, q \in A$. There are disjoint open sets $U$ and $V \in \tau$ containing $p$ and $q$, respectively. Let $U_{0}=(\beta f)^{-1}[U] \cap X$ and $V_{0}=(\beta f)^{-1}[V] \cap X$. Since $g^{-1}\left[U_{0}\right]=(\beta f)^{-1}[U]$ and $g^{-1}\left[V_{0}\right]=(\beta f)^{-1}[V]$, it follows that $U_{0}$ and $V_{0} \in \sigma$. But $U_{0}$ and $V_{0}$ are disjoint open sets containing $p$ and $q$, respectively. This shows that $(X, \sigma)$ is Hausdorff and completes the proof that $X$ has a compact subtopology.

We observe that if $X$ is a Tychonoff space and $R(X)$ is a retract of $X$ and $R(X)$ has a compact subtopology, then $X$ has a compact subtopology. Next, we focus our attention on countable subspaces. First, recall that a space is scattered if every nonempty subspace has an isolated point. In 1948, Katetov $[\mathbf{K}]$ proved that a regular, countable space has a compact subtopology iff it is scattered. We do not know if a countable, scattered space is Katetov but can prove the converse.

\subsection{TheOREM. A countable, Katetov space is scattered.}

ProOF. Let $Y$ be a countable, Katetov space. Then there is a continuous bijection $f: Y \rightarrow X$ where $X$ is minimal Hausdorff. If $A \subseteq Y$ and $f[A]$ has an isolated point, it follows that $A$ has an isolated point. So, it suffices to show that a countable, minimal Hausdorff space $X$ is scattered. First observe that a consequence of 1.6 is that a countable, minimal Hausdorff space has a dense set of isolated points. Now, we inductively construct a family $\left\{X_{\alpha}: \alpha<\omega_{1}\right\}$ of closed subsets of $X$ and a family $\left\{A_{\alpha}: \alpha<\omega_{1}\right\}$ of compact subsets of $E X$ such that for $\alpha<\omega_{1}$, (i) $k_{\alpha}: A_{\alpha} \rightarrow X_{\alpha}$ is a perfect irreducible surjection where $k_{\alpha}=k_{X} \mid A_{\alpha}$, (ii) $X_{\alpha} \backslash X_{\alpha+1}$ is a subset of isolated points of $X_{\alpha}$, (iii) $X_{\alpha} \backslash X_{\alpha+1} \neq \varnothing$ if $X_{\alpha} \neq \varnothing$, 
and (iv) for $\alpha<\beta<\omega_{1}, A_{\beta} \subseteq A_{\alpha}$. We start the induction by defining $X_{0}=X$ and $A_{0}=E X$. Suppose $\alpha<\omega_{1}$ and $\left\{X_{\beta}: \beta<\alpha\right\}$ and $\left\{A_{\beta}: \beta<\alpha\right\}$ are defined satisfying (i)-(iv). If $\alpha=\beta+1$ for some $\beta$, then as $k_{\beta}$ : $A_{\beta} \rightarrow X_{\beta}$ is a perfect irreducible surjection, $X_{\beta}$ is a Katetov space by 1.2. Let $X_{\beta}^{\prime}$ be $X_{\beta}$ with the coarser minimal Hausdorff topology; so, $k_{\beta}: A_{\beta} \rightarrow X_{\beta}^{\prime}$ is perfect, irreducible, $\theta$ continuous, and onto. For $p \in A_{\beta}$, it follows that $p$ is isolated in $A_{\beta}$ iff $k_{\beta}(p)$ is isolated in $X_{\beta}^{\prime}$. Now, $X_{\beta}^{\prime}$ has a dense set $D_{\beta}$ of isolated points. Let $X_{\alpha}=X_{\beta} \backslash D_{\beta}$ and $B_{\alpha}=A_{\beta} \backslash k_{\beta}^{-1}\left[D_{\beta}\right]$. Now, $B_{\alpha}$ is compact and there is a compact subspace $A_{\alpha}$ of $B_{\alpha}$ such that $k_{\beta} \mid A_{\alpha}: A_{\alpha} \rightarrow X_{\alpha}$ is a perfect, irreducible surjection; let $k_{\alpha}=k_{\beta} \mid A_{\alpha}$.

If $\alpha$ is a limit ordinal, then $k_{X}\left[\bigcap\left\{A_{\beta}: \beta<\alpha\right\}\right]=\bigcap\left\{X_{\beta}: \beta<\alpha\right\}$. Let $X_{\alpha}=$ $\bigcap\left\{X_{\beta}: \beta<\alpha\right\}$. There is a compact subspace $A_{\alpha}$ of $\bigcap\left\{A_{\beta}: \beta<\alpha\right\}$ such that $k_{X} \mid A_{\alpha}: A_{\alpha} \rightarrow X_{\alpha}$ is a perfect, irreducible surjection. This completes the proof of the induction. Since $\left\{X_{\alpha} \backslash X_{\alpha+1}: \alpha<\omega_{1}\right\}$ are pairwise disjoint, it follows that for some $\alpha<\omega_{1}, X_{\alpha} \backslash X_{\alpha+1}=\varnothing$ implying $X_{\alpha}=\varnothing$ by (iii). Thus $X$ is a scattered space.

Our next result is an example of a Cech-complete extremally disconnected space which is not Katetov. Recall (see [GJ]) that in an extremally disconnected space, dense subspaces are extremally disconnected and countable subspaces are $C^{*}$ embedded, convergent sequences are eventually constant and in a compact, extremally disconnected space, nonempty open and infinite closed subsets are uncountable and co-countable subspaces are dense.

5.3. THEOREM. Let $Y$ be a compact, extremally disconnected space without isolated points, and let $S=\left\{x_{n}: n \in \omega\right\}$ be a discrete subspace of $Y$. Then $X=Y \backslash S$ is not Katetov.

Proof. Since $X$ is dense in $Y, X$ is extremally disconnected. Assume, by way of contradiction, that $X$ has a coarser minimal Hausdorff topology $\tau$. Let $X^{\prime}=(X, \tau)$. For each $p \in X$, let $A_{p}=\bigcap\left\{\operatorname{cl}_{Y} U: p \in U \in \tau\right\}$. If $p, q \in X$ and $p \neq q$, there are disjoint open sets $U$ and $V$ in $\tau$ such that $p \in U$ and $q \in V$. Since $U, V \in \tau(X), X$ is dense in $Y$ and $Y$ is extremally disconnected, $\operatorname{cl}_{Y} U \cap \operatorname{cl}_{Y} V=\varnothing$. So, $A_{p} \cap A_{q}=\varnothing$ and $\left\{A_{p} \cap S: p \in X\right\}$ is a partition of the countable set $S$. Since $A_{p} \subseteq S \cup\{p\}, A_{p}$ is compact and $S \cup\{p\}$ is countable, it follows that $A_{p}$ is finite. Then $T=\left\{p \in X: A_{p} \neq\{p\}\right\}$ is countable; let $T=\left\{y_{n}: n \in \omega\right\}$. If $p \in X \backslash T$, then $A_{p}=\{p\}=\bigcap\left\{\operatorname{cl}_{Y} U: p \in U \in \tau\right\}$. Since $Y$ is compact, it follows that $\left\{\operatorname{cl}_{Y} U: p \in U \in \tau\right\}$ is a neighborhood base of $p$ in $Y$. So, id: $X \backslash T \rightarrow X^{\prime} \backslash T$ is a homeomorphism. Since $T$ is countable, $X^{\prime} \backslash T=X \backslash T=Y \backslash S \cup T$ is extremally disconnected. So, $E\left(X^{\prime} \backslash T\right)=X \backslash T$. Hence $X^{\prime}$ is a minimal Hausdorff extension of $X^{\prime} \backslash T$ and $E\left(X^{\prime}\right)=\beta E\left(X^{\prime} \backslash T\right)=\beta(X \backslash T)=Y$. Thus, there is a perfect, irreducible $\Theta$-continuous surjection $f: Y \rightarrow X^{\prime}$ such that $f(x)=x$ for $x \in X \backslash T$. It is easy to show that $f(x)=x$ for all $x \in X$. Let $z_{n}=f\left(x_{n}\right)$ for $n \in \omega$, and $R=\left\{z_{n}: n \in \omega\right\}$. So, $f[S]=R$ and $f[R]=R$ as $f \mid X=$ id. Since $f\left[\operatorname{cl}_{Y} S\right]$ is closed, $\operatorname{cl}_{X^{\prime}} R \subseteq f\left[\operatorname{cl}_{Y}(S)\right]=R \bigcup\left(\operatorname{cl}_{Y} S \backslash S\right)$. Thus, $\operatorname{cl}_{X} R \subseteq \operatorname{cl}_{X^{\prime}} R \subseteq R \cup \operatorname{cl}_{Y} S \backslash S$. There are two possibilities.

Case 1. $R \backslash \operatorname{cl}_{Y} S$ is finite. As $S$ is discrete in $Y$ and $C^{*}$-embedded in $Y, \operatorname{cl}_{Y} S \backslash S$ is homeomorphic to $\beta \omega \backslash \omega$. Since $\operatorname{cl}_{X^{\prime}} R \subseteq R \cup\left(\mathrm{cl}_{Y} S \backslash S\right)$, it follows that $\mathrm{cl}_{X^{\prime}} R$ is compact. Since $\beta \omega \backslash \omega$ is not separable, there is a point $p \in\left(\operatorname{cl}_{Y} S \backslash S\right) \backslash \operatorname{cl}_{X^{\prime}} R$. There is an open set $U \in X^{\prime}$ such that $p \in U$ and $\operatorname{cl}_{X^{\prime}} U \cap \operatorname{cl}_{X^{\prime}} R=\varnothing$. Since $f$ is 
$\Theta$-continuous, there is an open set $V$ in $Y$ such that $p \in V$ and $f\left[\operatorname{cl}_{Y} V\right] \subseteq \operatorname{cl}_{X^{\prime}} U$. Since $p \in \operatorname{cl}_{Y} S \backslash S, V \cap S \neq \varnothing$. But $\varnothing \neq f[V \cap S] \subseteq R$ leads to a contradiction as $f[V \cap S] \subseteq \mathrm{cl}_{X^{\prime}} U$ and $\operatorname{cl}_{X^{\prime}} U \cap R=\varnothing$.

Case 2. $R \backslash \mathrm{cl}_{Y} S$ is infinite. First, we will find an infinite discrete subspace $P \subseteq R \backslash \operatorname{cl}_{Y} S$ such that $\operatorname{cl}_{Y} P \cap S=\varnothing$. Since $S$ is discrete in $Y$, there is a family $\left\{V_{n}: n \in \omega\right\}$ of pairwise disjoint open sets such that $x_{n} \in V_{n}$ for $n \in \omega$. If $M=$ $\left\{n \in \omega:\left(R \backslash \operatorname{cl}_{Y} S\right) \cap V_{n} \neq \varnothing\right\}$ is infinite, let $p_{n} \in\left(R \backslash \operatorname{cl}_{Y} S\right) \cap V_{n}$ for $n \in M$; then $P=\left\{p_{n}: n \in M\right\}$ is an infinite discrete subspace of $R \backslash \operatorname{cl}_{Y} S$ and $\operatorname{cl}_{Y} P \cap S=\varnothing$. If $M$ is finite, there is some $n \in \omega$ such that $Q=V_{n} \cap\left(R \backslash \operatorname{cl}_{Y} S\right)$ is infinite. Since no sequence in $Q$ converges to $x_{n}$, there is an open set $U \subseteq V_{n}$ such that $x_{n} \in U$ and $Q \backslash U$ is infinite. Let $P$ be an infinite discrete subspace of $Q \backslash U$. Then $P \subseteq R \backslash \operatorname{cl}_{Y} S$ and $\operatorname{cl}_{Y} P \cap S=\varnothing$. Since $S \cup P$ is $C^{*}$-embedded in $Y$ and $S$ and $P$ are completely separated in $S \cup P$, then $\operatorname{cl}_{Y} S \cap \operatorname{cl}_{Y} P=\varnothing$. Now, $\operatorname{cl}_{Y} P \subseteq \operatorname{cl}_{Y} R \subseteq \operatorname{cl}_{X} R \cup S \subseteq$ $R \cup \mathrm{cl}_{Y} S$. So, $\operatorname{cl}_{Y} P \subseteq R$, which is impossible as $\operatorname{cl}_{Y} P$ is uncountable.

Since both cases lead to contradictions, our original assumption that $X$ is Katetov is false.

Finally, we present an example of a zero-dimensional space $X$ with a compact subtopology such that $E X$ is not Katetov.

5.4. EXAMPLE. Let $Y$ be a compact extremally disconnected space without isolated points, $S=\left\{x_{n}: n \in \omega\right\}$ be a countable discrete subset of $Y$ and $T=Y \backslash S$. Let $X$ be the quotient space of $T$ where the compact, nowhere dense set $\operatorname{cl}_{Y} S \backslash S$ is identified to a point. It follows that $X$ is zero-dimensional, $X$ is locally compact except for one point and the quotient function from $T$ to $X$ is perfect, irreducible and continuous. By 2.7, $X$ has a compact subtopology. Also, $E X=T$ is not Katetov by 5.3 .

\section{REFERENCES}

[BPS] M. P. Berri, J. R. Porter and R. M. Stephenson, Jr., A survey of minimal topological spaces, General Topology and its Relations to Modern Analysis and Algebra, III (Proc. Conf. Kanpur, 1968), Academia, Prague, 1971, pp. 93-114.

[BL] A. Blasczyk and U. Lorek, A classification of $H$-closed extensions, Colloq. Math. 39 (1978), 29-33.

[B] N. Bourbaki, Espaces minimaux et espaces complètement sèparès, C. R. Acad. Sci. Paris 212 (1941), 215-218.

[DP 1] R. F. Dickman, Jr., and J. R. Porter, $\Theta$-closed subsets of Hausdorff spaces, Pacific J. Math. 59 (1975), 407-415.

[DP $\left.\mathbf{P}_{\mathbf{2}}\right]$ A. Dow and J. R. Porter, Cardinalities of $H$-closed spaces, Topology Proc. 7 (1982), 27-50.

[D] J. Dugundji, Topology, Allyn \& Bacon, Boston, Mass., 1967.

[E] R. Engelking, General topology, Polish Scientific Publishers, Warszawa, 1977.

[F] S. Fomin, Extensions of topological spaces, Ann. of Math. (2) 44 (1943), 471-480.

[GJ] L. Gillman and M. Jerison, Rings of continuous functions, Van Nostrand Reinhold, New York, 1960.

[G] A. A. Gryzlov, On Katetov spaces, Soviet Math. (Iz. VUZ) 91 (1975), 14-18.

$\left[\right.$ He] H. Herrlich, $T_{\nu}$-Abgeschlossenheit und $T_{\nu}$-Minimalität, Math. Z. 88 (1965), 285-294.

[Ho $]$ T. Hoshina, Countable-points compactifications for metric spaces, Fund. Math. 103 (1979), 123132.

[Ho2] _ Countable-points compactifications of product spaces, Tsukuba J. Math. 6 (1982), 231-236.

[K] M. Katetov, On mappings of countable spaces, Colloq. Math. 2 (1948), 30-33.

[Pa] A. Parhomenko, On one-to-one continuous functions, Mat. Sb. 5 (1939), 197-210.

[Po] J. R. Porter, Lattices of $H$-closed extensions, Bull. Acad. Polon. Sci. Sér. Sci. Math. Astronom. Phys. 22 (1974), 831-837. 
[PV $]$ J. R. Porter and C. Votaw, H-closed extensions. I, General Topology Appl. 3 (1973), 211-224. [PV $\left.\mathbf{P V}_{2}\right], H$-closed extensions. II, Trans. Amer. Math. Soc. 202 (1975), 193-209.

[Py] E. G. Pytkeev, Upper bounds of topologies, Math. Notes 20 (1976), 831-837.

[R] L. Rudolf, Minimal Hausdorff spaces are pseudo-quotient images of compact Hausdorff ones, Colloq. Math. Soc. János Bolyai 8, Topics in Topology, Keszthely, Hungary, 1972, pp. 563-567.

[S] D. P. Strauss, Extremally disconnected spaces, Proc. Amer. Math. Soc. 18 (1967), 305-309.

[V1] J. Vermeer, Expansions of $H$-closed spaces, Doctoral Dissertation, Free University, Amsterdam, 1983.

[V $\left.\mathrm{V}_{2}\right]$, Closed subspaces of $H$-closed spaces, Pacific J. Math. 118 (1985).

[VW] J. Vermeer and E. Wattel, Projective elements in categories with perfect $\Theta$-continuous maps, Canad. J. Math. 23 (1981), 872-884.

[Wh] G. T. Whyburn, Directed families of sets and closedness of functions, Proc. Nat. Acad. Sci. U.S.A. 54 (1965), 688-692.

[Wo] R. G. Woods, A survey of absolutes of topological spaces, Top. Structures II, Math. Centre Tracts no. 116, Math. Centrum, Amsterdam, 1979, pp. 323-362.

DePARTMENT OF MATHEMATICS, UNIVERSity OF KANSAS, LAWRENCE, KANSAS 66045 